\title{
Plasma concentrations of leptin at mid- pregnancy are associated with gestational weight gain among pregnant women in Tanzania: a prospective cohort study
}

Dongqing Wang ${ }^{1 *}$, Anne Marie Darling ${ }^{1}$, Chloe R. McDonald ${ }^{2}$, Nandita Perumal', Enju Liu ${ }^{3}$, Molin Wang ${ }^{4,5}$, Said Aboud ${ }^{6}$, Willy Urassa ${ }^{6}$, Andrea L. Conroy ${ }^{7}$, Kyla T. Hayford ${ }^{8}$, W. Conrad Liles ${ }^{9}$, Kevin C. Kain ${ }^{10}$ and Wafaie W. Fawzi ${ }^{1,5,11}$

\begin{abstract}
Background: Gestational weight gain (GWG) has critical implications for maternal and child health. Inflammation and angiogenesis are implicated in various aspects of maternal metabolism that may play a role in gestational weight gain. The associations of inflammatory, angiogenic, and metabolic pathways with GWG are yet to be elucidated. This study evaluated associations between a panel of inflammatory, angiogenic, and metabolic proteins measured in midpregnancy and gestational weight gain.

Methods: Pregnant women were enrolled from Dar es Salaam, Tanzania, between 2001 and 2004. The participants were enrolled at mid-pregnancy (12 to 27 weeks of gestation) and followed up until delivery. This analysis focused on a cohort of 1002 women who were primigravid, had singleton live births, had longitudinal measures of gestational weight, and whose mid-pregnancy plasma samples underwent analysis for 18 proteins.
\end{abstract}

Results: Higher plasma concentrations of leptin (mean difference in GWG percent adequacy comparing highest with lowest quartiles: $10.24 ; 95 \% \mathrm{Cl} 3.31,17.16 ; p$-trend $=0.003$ ) and chitinase-3-like protein-1 (CH3L1) (mean difference in GWG percent adequacy comparing highest with lowest quartiles: $7.02 ; 95 \% \mathrm{Cl} 0.31,13.72 ; p$-trend $=0.007$ ) were associated with greater GWG in a dose-response pattern. Higher leptin concentrations were associated with a lower risk of inadequate GWG (risk ratio comparing highest with lowest quartiles: $0.77 ; 95 \% \mathrm{Cl} 0.65,0.91 ; p$-trend $=0.001$ ) and a higher risk of excessive GWG (risk ratio comparing highest with lowest quartiles: 1.57; $95 \% \mathrm{Cl} 1.03,2.39$; $p$-trend $=0.03$ ). Higher $\mathrm{CH} 3 \mathrm{~L} 1$ concentrations were associated with a higher risk of excessive GWG ( $p$-trend $=0.007)$. The associations of leptin and CH3L1 with inadequate GWG were stronger during the second than the third trimester. The other 16 proteins examined were not significantly associated with GWG.

Conclusions: Mid-pregnancy plasma leptin concentrations may be associated with GWG and have clinical predictive utility in identifying women at a higher risk of inadequate or excessive gestational weight gain.

\footnotetext{
*Correspondence: dqwang@hsph.harvard.edu

${ }^{1}$ Department of Global Health and Population, Harvard T.H. Chan School

of Public Health, Harvard University, Boston, MA 02120, USA

Full list of author information is available at the end of the article
} original author(s) and the source, provide a link to the Creative Commons licence, and indicate if changes were made. The images or other third party material in this article are included in the article's Creative Commons licence, unless indicated otherwise in a credit line to the material. If material is not included in the article's Creative Commons licence and your intended use is not permitted by statutory regulation or exceeds the permitted use, you will need to obtain permission directly from the copyright holder. To view a copy of this licence, visit http://creativecommons.org/licenses/by/4.0/. The Creative Commons Public Domain Dedication waiver (http://creativeco mmons.org/publicdomain/zero/1.0/) applies to the data made available in this article, unless otherwise stated in a credit line to the data. 
Keywords: Pregnancy, Weight gain during pregnancy, Gestational weight, Inflammatory proteins, Metabolic proteins, Tanzania, Sub-Saharan Africa, Leptin, CH3L1, Biomarkers

\section{Background}

Gestational weight gain (GWG) is a complex process that supports the functions of growth and development of the fetus during pregnancy [1], with important implications for maternal and child health. On the one hand, inadequate GWG increases the risks of low birthweight [1-3], preterm birth [1, 3, 4], small for gestational age $[1,2,4]$, and fetal and neonatal death $[5$, 6]. On the other hand, excessive GWG is a risk factor for maternal morbidity [7], large for gestational age [1, $4]$ and postpartum weight retention [1, 2, 8]. Emerging evidence indicates that excessive GWG also increases the risk of the offspring being overweight or obese in their childhood and adulthood [8-12]. GWG is an essential target for antenatal monitoring [13] and preconceptional care [14]. In 2009, the National Academy of Medicine, formerly the Institute of Medicine (IOM), released guidelines on the recommended weight gain during pregnancy [1].

Accumulating evidence suggests that many women in low- and middle-income countries experience inadequate GWG. The mean level of GWG in sub-Saharan Africa has been estimated to be only 50 to $60 \%$ of the amount recommended by the IOM [15-17]. Pregnant women in the United Republic of Tanzania have a mean GWG level of $5.5 \mathrm{~kg}$, which is less than $50 \%$ of the minimum recommendation $(11.5 \mathrm{~kg}$ in normalweight women and $12.5 \mathrm{~kg}$ in underweight women) and is among the lowest in sub-Saharan Africa [17]. At the same time, there is an emerging burden of excessive GWG in resource-constrained countries [16], which is likely to continue increasing with the nutritional transition to more Western diets, the increasingly sedentary lifestyles, and the rising tide of overweight and obesity $[18,19]$.

GWG is simultaneously affected by various maternal characteristics, including demographics, reproductive history, dietary intake, physical activity, psychological factors, and pre-existing health conditions [1]. Inflammatory and angiogenic processes and crosstalk between these pathways may also play a role in the accumulation of weight during pregnancy. However, the associations between inflammatory, angiogenic, and metabolic proteins and GWG remain poorly elucidated, especially in sub-Saharan Africa, where inflammation induced by common infections remains high and pregnant women are at a greater risk of poor birth outcomes [20].
Assessing the associations of inflammatory, angiogenic, and metabolic proteins with GWG may shed light on the mechanisms that contribute to GWG and reveal potential markers for antenatal monitoring of inadequate or excessive GWG. In this study, we evaluated associations of a panel of proteins measured in mid-pregnancy with GWG adequacy levels in Tanzania. We also aimed to explore whether potential associations may be modified by maternal characteristics.

\section{Methods}

\section{Study design and study population}

This study is a secondary analysis of a randomized, double-blind, placebo-controlled trial of maternal multiple micronutrient supplementation (MMS) in Tanzania. The primary aim of the study was to assess the impacts of prenatal MMS on fetal loss, preterm birth, and low birthweight. The design and findings of the parent study have been described elsewhere [21]. Briefly, pregnant women who attended antenatal clinics in Dar es Salaam, Tanzania were enrolled between August 2001 and July 2004. The eligibility criteria included: 1 ) negative for HIV infection based on HIV-1 serologic status [22]; 2) gestational age (GA) between 12 and 27 weeks at enrollment, based on the date of last menstrual period; 3) maternal age of 18 years or older; and 4) an intention to deliver and stay in Dar es Salaam for at least 1 year after delivery. From enrollment through delivery, the women were randomly assigned to receive daily oral MMS or placebo. The study enrolled 8428 eligible women, of whom 6 died before delivery and 43 were lost to follow-up by the time of delivery. Among the remaining 8379 women, 8223 were pregnant with singletons. This study was approved by the institutional review boards at Muhimbili University of Health and Allied Sciences, the University Health Network (Toronto, Ontario, Canada), and Harvard T.H. Chan School of Public Health. All women provided written informed consent to participate.

\section{Data collection}

Participants had study visits by medical providers monthly until 32 weeks of gestation, then every 2 weeks until 36 weeks of gestation, and then weekly until 6 weeks after delivery. At baseline enrollment, research nurses used a questionnaire to collect data on demographic characteristics, socioeconomic status, reproductive and medical history, and behavioural factors, 
including smoking and alcohol consumption. Household ownership of five assets, including sofa or couch, television, radio, refrigerator, and fan, was collected at baseline using a questionnaire, A baseline wealth index was constructed based on household asset ownership using principal component analysis [23], and the same index has been used in previous publications of this cohort [24-27]. At baseline, research nurses measured maternal height to the nearest $0.1 \mathrm{~cm}$ using a stadiometer with headcovers and shoes removed. Weight was measured at baseline and every follow-up visit to the nearest $100 \mathrm{~g}$ using balanced scales with the participant wearing light clothing without shoes. Trained research assistants obtained information on dietary intake using monthly 24-h recalls until 36 weeks of gestation.

Maternal peripheral blood samples were collected in EDTA vacutainer tubes at enrollment. The plasma samples underwent analysis for 18 inflammatory, angiogenic, and metabolic proteins among a random subset of 1078 women who were primigravid, had singleton live births, and had stored plasma samples available [26]. The measurements of the proteins have been described elsewhere [20, 26]. Briefly, the 18 proteins included 1) angiopoietin-1 (Ang-1); 2) angiopoietin-2 (Ang-2); 3) angiopoietin-like 3 (Angptl3); 4) vascular endothelial growth factor (VEGF-A); 5) soluble fms-like tyrosine kinase 1 (sFlt-1); 6) soluble tumor necrosis factor receptor 2 (sTNFR2); 7) placental growth factor (PGF); 8) macrophage inflammatory protein-1 beta (MIP $\beta / C C L 4)$; 9) monocyte chemoattractant protein-1 (MCP-1/CCL2); 10) leptin; 11) interleukin-1 beta (IL-1 $\beta)$; 12) interleukin-18 binding protein (IL-18 BP); 13) soluble intercellular adhesion molecule-1 (sICAM1); 14) complement factor D (Factor D); 15) soluble endoglin (sEng); 16) C-reactive protein (CRP); 17) chitinase-3-like protein-1 (CHI3L1); and 18) complement component C5a (C5a). We selected this panel of proteins based on previous literature and work in our laboratory indicating their associations with pregnancy physiology and adverse birth outcomes [28-34].

\section{Statistical analysis}

\section{Estimation of early-pregnancy weight}

As the participants were 12 to 27 weeks of gestation at enrollment, $97.7 \%$ of the participants did not have an observed weight measure during the first trimester of pregnancy. An accurate assessment of GWG requires a pre-pregnancy weight measure or a measure of first-trimester weight as a proxy [35]. We have previously developed an approach to imputing first-trimester weight. The development and validation of this approach and the comparison with alternative strategies have been described in detail elsewhere [35]. Briefly, this approach employed mixed-effects models with restricted cubic splines to estimate the gestational weight in the first trimester using longitudinal weight measures collected later during pregnancy. We imputed the weight at $9^{0 / 7}$ weeks instead of another time point (e.g., prepregnancy weight) to avoid undue extrapolation. As weight gain during the first trimester is minimal [1], the weight imputed close to the midpoint of the first trimester served as a reasonable proxy for the pre-pregnancy weight.

First-trimester body mass index (BMI) was calculated by dividing first-trimester weight (observed during the first trimester or imputed at $9^{0 / 7}$ weeks) in kilograms by the square of height in meters. For women aged $\geq 19$ years old, we used the cutoffs by the World Health Organization (WHO) to define underweight (BMI: < 18.5), normal weight (BMI: 18.5 to $<25.0$ ), overweight (BMI: 25.0 to $<30.0$ ), and obesity (BMI: $\geq 30.0$ ). For adolescent women (i.e., less than 20 years old based on the United Nations' definition), we used the WHO growth reference to define underweight (BMI-for-age Z-score: < $-2 \mathrm{SD}$ ), normal weight (BMI-for-age Z-score: $-2 \mathrm{SD}$ to $<1 \mathrm{SD}$ ), overweight (BMI-for-age Z-score: $1 \mathrm{SD}$ to $<2 \mathrm{SD}$ ), and obese (BMI-for-age Z-score: $\geq 2 \mathrm{SD}$ ) [36].

\section{Metrics of gestational weight gain}

GWG was quantified as the percent adequacy compared to the weight gain recommended by the IOM. First, we calculated the total GWG (in kilograms) for each participant as the difference between the last available weight during pregnancy and the first-trimester weight (imputed or observed). Second, we estimated the IOM-recommended GWG (in kilograms) for each woman at the time when the last weight measure was taken. Finally, the GWG percent adequacy was calculated by dividing the observed GWG by the recommended GWG at the last weight measurement. The overall formula for the GWG percent adequacy is displayed below.

$$
\begin{aligned}
\text { GWG percent adequacy }(\%) & =\frac{\text { Observed GWG }}{\text { Recommended GWG }} \times 100 \\
& =\frac{\text { last available weight }- \text { imputed T1 weight }}{\text { expected T1 GWG }+(\text { GA at last available weight }-13.86 \text { wks }) \times \text { recommended weekly rate of GWG for T2 \& T3 }} \times 100
\end{aligned}
$$


The GWG percent adequacy has been calculated in the same way in previous studies [37]. This metric accounts for the different gestational durations at last weight measurements and takes advantage of well-established recommendations. In addition to the continuous measure of percent adequacy, we created binary outcomes that correspond to inadequate, adequate, and excessive GWG. We defined inadequate GWG as percent adequacy $<90 \%$, adequate GWG as percent adequacy of 90 to $125 \%$, and excessive GWG as percent adequacy $>125 \%$, consistent with previous literature [37]. We also calculated the trimester-specific weekly rates of weight gain using the first and last available measures within the second and third trimesters; inadequate, adequate, and excessive weekly GWG were defined similarly based on the rate recommended by the IOM [1].

\section{Regression analyses}

The regression analyses included 1002 primigravid women with singleton live births who had data available on plasma concentrations of the proteins (which was measured in a random subset of all women with singleton live births), longitudinal gestational weight measures, and key potential confounders (education level, marital status, and wealth index). The plasma concentrations of each protein were divided into quartiles based on the distributions in the analytical sample. We used linear models to evaluate the associations of the proteins with the continuous metric of GWG percent adequacy and reported the mean differences and 95\% confidence intervals (CIs), comparing each quartile to the lowest quartile. We used log-binomial models to evaluate the associations of the proteins with the binary metrics of GWG, including inadequate GWG and excessive GWG. We used modified Poisson models with robust variance estimation to handle model convergence issues whenever necessary $[38,39]$. We reported the risk ratios (RRs) and 95\% CIs comparing each quartile to the lowest quartile.

All models were adjusted for potential confounders, including maternal age at enrollment, maternal education level, marital status, maternal occupation, household wealth index, total energy intake, and first-trimester BMI category. We also adjusted for the randomly assigned intervention (MMS or control) as prenatal MMS has been previously shown to increase GWG in this cohort of women [27].

For proteins associated with GWG in the overall sample, we additionally included cross-product terms and conducted subgroup analyses to explore effect modification by the following four maternal characteristics: 1) prenatal regimen (MMS or control); 2) first-trimester BMI category (underweight, normal-weight, or overweight/ obese); 3) maternal anaemia status at enrollment defined as any anaemia (haemoglobin $<11 \mathrm{~g} / \mathrm{dL}$ ) or no anaemia [40]; and 4) maternal stature $(<150 \mathrm{~cm}$ or $\geq 150 \mathrm{~cm})$. All analyses were conducted using SAS 9.4 (SAS Institute Inc., Cary, North Carolina) with a two-sided $\alpha$ level of 0.05. We did not adjust for multiple testing [41].

\section{Results}

General characteristics at enrollment and GWG outcomes of the pregnant women are presented in Table 1, and their mid-pregnancy concentrations of the proteins are presented in Table 2. At baseline, the participants were on average 22 years (range: 18 to 41 years) of age and at 21 weeks (range: 12 to 27 weeks) of gestation. All participants included in this analysis were primigravida and pregnant with a singleton fetus. Over $90 \%$ of the sample had 5 or more years of education, nearly $79 \%$ were married or cohabiting, and approximately $78 \%$ were unemployed. Approximately 12, 75, 11, and 2\% of the participants were underweight, normal-weight, overweight, and obese, respectively; the mean BMI during early pregnancy was $21.8 \mathrm{~kg} / \mathrm{m}^{2}$ (SD: $3.27 \mathrm{~kg} / \mathrm{m}^{2}$ ). The median GWG percent adequacy was $88.3 \%$ (25th percentile, 75th percentile: 68.8, 112.3\%). Approximately 53,30 , and $17 \%$ of the participants had inadequate, adequate, and excessive GWG, respectively.

Higher mid-pregnancy plasma leptin concentrations were associated with a greater GWG percent adequacy (Table 3). Participants in the third and fourth quartiles of leptin concentrations had 6.9\% (95\% CI 0.12, 13.66) and $10.2 \%$ (95\% CI 3.31, 17.16) greater GWG percent adequacy, respectively, compared with those in the lowest quartile $(p$-trend $=0.003)$. Higher mid-pregnancy plasma CHI3L1 concentrations were also associated with a greater GWG percent adequacy. Participants in the highest quartile of CHI3L1 had 7.0\% (95\% CI 0.31, 13.72) greater GWG percent adequacy compared with women in the lowest quartile $(p$-trend $=0.007)$. The other 16 proteins examined were not associated with GWG percent adequacy.

Higher mid-pregnancy plasma leptin concentrations were associated with a lower risk of inadequate GWG and a higher risk of excessive GWG (Table 4). Participants in the highest quartile of leptin had 23\% $(R R=0.77 ; 95 \%$ CI $0.65,0.91$ ) lower risk of inadequate GWG compared with women in the lowest quartile $(p$-trend $=0.001)$. Participants in the highest quartile of leptin had a $57 \%$ $(R R=1.57$; 95\% CI 1.03, 2.39) higher risk of excessive GWG compared with women in the lowest quartile ( $p$-trend $=0.03$ ). Higher mid-pregnancy plasma CHI3L1 concentrations were associated with a higher risk of excessive GWG ( $p$-trend=0.007), although none of the quartiles reached statistical significance. Analyses on the trimester-specific weekly rate of GWG showed that the 
Table 1 Maternal characteristics at enrollment and gestational weight gain outcomes in a cohort of pregnant women in Dar es Salaam, Tanzania, 2001-2004

\begin{tabular}{|c|c|}
\hline \multicolumn{2}{|l|}{ Maternal characteristics } \\
\hline Number of women, $N$ & 1002 \\
\hline Age at enrollment, years & $21.8(3.17)$ \\
\hline Gestational age at enrollment, weeks & $21.3(3.55)$ \\
\hline \multicolumn{2}{|l|}{ Maternal education in years, $\%$} \\
\hline 0 to 4 & $83(8.3)$ \\
\hline 5 to 7 & $659(65.8)$ \\
\hline 8 to 11 & $214(21.4)$ \\
\hline$\geq 12$ & $46(4.6)$ \\
\hline \multicolumn{2}{|l|}{ Marital status, \% } \\
\hline Married/cohabiting & $789(78.7)$ \\
\hline Single/divorced/widowed & $213(21.3)$ \\
\hline \multicolumn{2}{|l|}{ Maternal occupation, ${ }^{\text {, } \% ~}$} \\
\hline Unemployed & $762(78.5)$ \\
\hline Employed & $209(21.5)$ \\
\hline First-trimester BMI ${ }^{c}$ & $21.8(3.3)$ \\
\hline \multicolumn{2}{|l|}{ First-trimester BMI category, ${ }^{c} \%$} \\
\hline Underweight & $120(12.0)$ \\
\hline Normal weight & $749(74.8)$ \\
\hline Overweight & $115(11.5)$ \\
\hline Obese & $18(1.8)$ \\
\hline Total energy intake, ${ }^{d} \mathrm{kcal} / \mathrm{d}$ & $2278.0(840.6)$ \\
\hline \multicolumn{2}{|l|}{ Intervention assignment, \% } \\
\hline Multiple micronutrient supplementation & $523(52.2)$ \\
\hline Control & $479(47.8)$ \\
\hline \multicolumn{2}{|l|}{ Gestational weight gain } \\
\hline Gestational weight gain percent adequacy & $88.3(68.8,112.3)$ \\
\hline \multicolumn{2}{|c|}{ Category of gestational weight gain adequacy, ${ }^{e} \%$} \\
\hline Inadequate & $533(53.2)$ \\
\hline Adequate & $299(29.8)$ \\
\hline Excessive & $170(17.0)$ \\
\hline
\end{tabular}

a Values are mean (standard deviation) for normally distributed continuous variables, median (25th percentile, 75 th percentile) for continuous variables with skewed distributions, and count (percentage) for categorical variables. BMI, body mass index

${ }^{\mathrm{b}}$ Maternal occupation was missing for 31 women

c Based on the observed weight during the first trimester or imputed weight at $9^{0 / 7}$ weeks

${ }^{d}$ Total energy intake was calculated as the average intake during pregnancy based on multiple 24-h recalls; missing for 61 women

e Inadequate, adequate, and excessive gestational weight gain were defined as $<90,90$ to $125 \%$, and $>125 \%$ percent adequacy, respectively, based on the Institute of Medicine guidelines

associations of leptin and CHI3L1 with inadequate GWG were stronger during the second trimester than during the third trimester (Additional file 1). The other 16 proteins examined were not associated with inadequate or excessive GWG (Additional file 2).
Table 2 Mid-pregnancy plasma concentrations of inflammatory, angiogenic, and metabolic proteins in a cohort of pregnant women in Dar es Salaam, Tanzania, 2001-2004

\begin{tabular}{lll}
\hline Proteins & N & $\begin{array}{l}\text { Median }(25 \text { th } \\
\text { percentile, 75th } \\
\text { percentile) }\end{array}$ \\
\hline Ang-1, ng/mL & 1001 & $14.88(8.15,23.57)$ \\
Ang-2, ng/mL & 1001 & $4.63(2.01,9.21)$ \\
Angptl3, ng/mL & 996 & $87.37(56.49,130.85)$ \\
VEGF-A, pg/mL & 998 & $39.66(7.81,262.25)$ \\
sFlt-1, ng/mL & 967 & $1.27(0.55,3.03)$ \\
sTNFR2, ng/mL & 999 & $5.04(3.27,7.50)$ \\
PGF, ng/mL & 950 & $1.18(0.54,2.24)$ \\
MIPß/CCL4, pg/mL & 982 & $147.21(56.10,346.06)$ \\
MCP-1/CCL2, pg/mL & 990 & $75.29(7.81,868.80)$ \\
Leptin, ng/mL & 1001 & $8.39(4.66,13.79)$ \\
IL-13, pg/mL & 386 & $20.84(3.91,76.40)$ \\
IL-18 BP, ng/mL & 1002 & $12.98(8.74,19.03)$ \\
sICAM1, ng/mL & 1002 & $151.39(103.53,226.81)$ \\
Factor D, ng/mL & 377 & $487.32(326.87,681.80)$ \\
sEng, ng/mL & 1001 & $21.19(14.62,28.10)$ \\
CRP, $\mu$ g/mL & 372 & $1.87(0.81,4.14)$ \\
CHI3L1, ng/mL & 1002 & $37.54(22.75,66.69)$ \\
C5a, ng/mL & 991 & $79.20(30.65,236.78)$ \\
\hline
\end{tabular}

${ }^{a}$ Ang-1 angiopoietin-1; Ang-2 angiopoietin-2; Angpt/3 angiopoietin-like 3; C5a complement component C5a; CHI3L 1 chitinase-3-like protein-1; CRP C-reactive protein; Factor $D$ complement factor D; IL-18 BP interleukin-18 binding protein; $I L-1 \beta$ interleukin-1 beta; $M C P-1 / C C L 2$ monocyte chemoattractant protein-1; $M I P \beta / C C L 4$ macrophage inflammatory protein-1 beta; $P G F$ placental growth factor; sEng soluble endoglin; sFlt-1 soluble fms-like tyrosine kinase 1; sICAM1 soluble intercellular adhesion molecule-1; sTNFR2 soluble tumor necrosis factor receptor 2; VEGF-A vascular endothelial growth factor

Results from the exploratory analyses of interaction using cross-product terms are shown in Additional file 3. There were statistical interactions between leptin and first-trimester BMI category on GWG percent adequacy $(\mathrm{p}$-interaction $=0.02)$, between leptin and baseline maternal anaemia on inadequate GWG (p-interaction $=0.04$ ), and between leptin and maternal stature on excessive GWG (p-interaction $=0.02$ ). There were also statistical interactions between CHI3L1 and first-trimester BMI category on GWG percent adequacy (p-interaction $<0.001)$ and between CHI3L1 and first-trimester BMI category on inadequate GWG (p-interaction $=0.001$ ). Subgroup analyses of potential effect modification (Additional files 4 and 5) show that: 1) the positive associations of leptin and CHI3L1 with GWG percent adequacy were stronger among overweight or obese women than normal-weight and underweight women; 2) the inverse association of leptin with inadequate GWG was stronger among anaemic women than non-anaemic women; and 
Table 3 Mid-pregnancy plasma concentrations of inflammatory, angiogenic, and metabolic proteins and gestational weight gain percent adequacy in a cohort of pregnant women in Dar es Salaam, Tanzania, 2001-2004, b

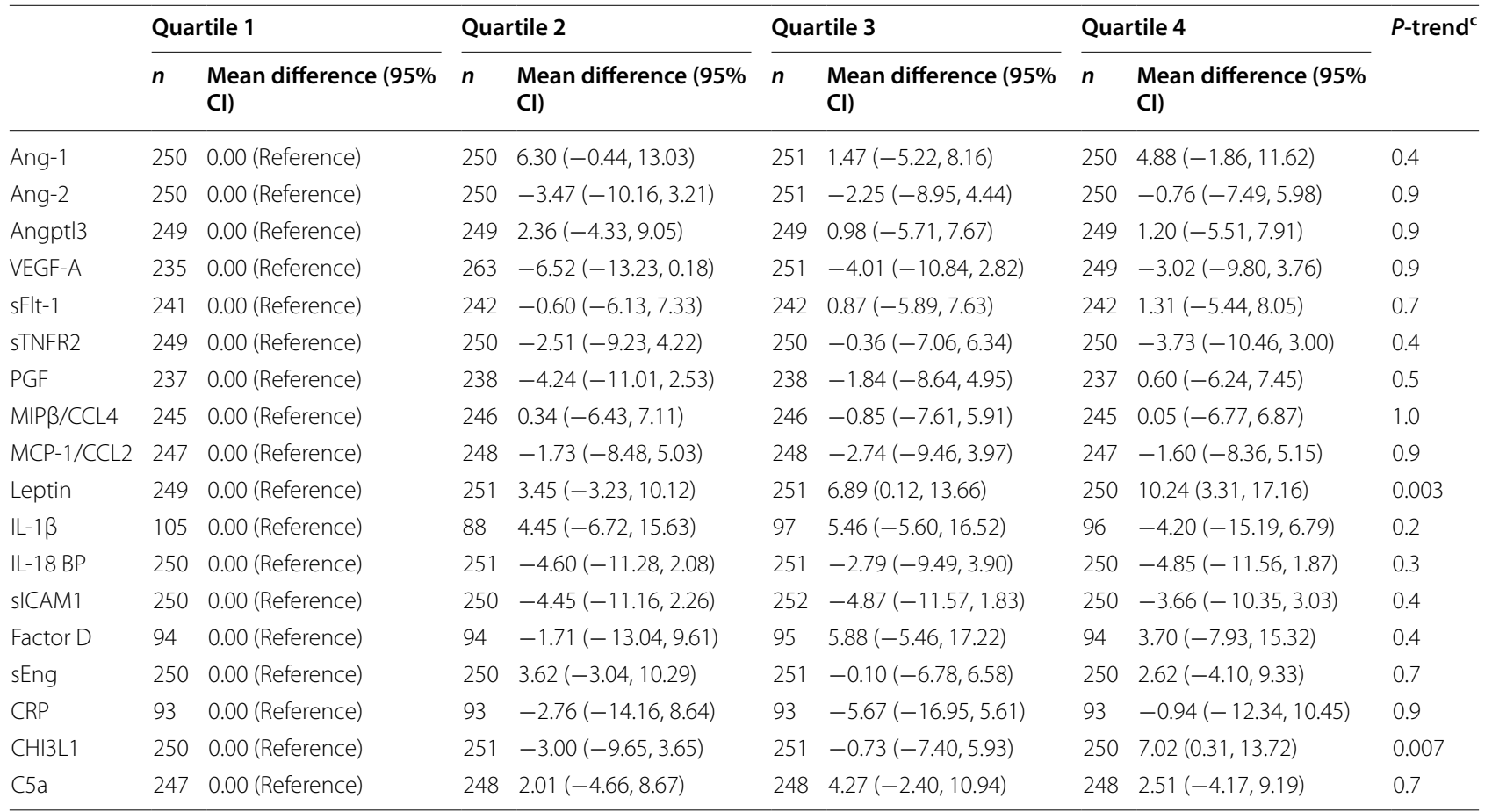

${ }^{a}$ Estimates were obtained from linear models comparing the upper three quartiles to the lowest quartile. Ang-1 angiopoietin-1; Ang-2 angiopoietin-2; Angpt/3 angiopoietin-like 3; $C 5 a$ complement component C5a; $C H I 3 L 1$ chitinase-3-like protein-1; $C l$ confidence interval; $C R P C$-reactive protein; Factor D complement factor D; IL-18 BP interleukin-18 binding protein; IL-1 $\beta$ interleukin-1 beta; $M C P-1 / C C L 2$ monocyte chemoattractant protein-1; $M I P \beta / C C L 4$ macrophage inflammatory protein-1 beta; PGF placental growth factor; sEng soluble endoglin; sFIt-1 soluble fms-like tyrosine kinase 1; sICAM1 soluble intercellular adhesion molecule-1; sTNFR2 soluble tumor necrosis factor receptor 2; VEGF-A vascular endothelial growth factor

b All models were adjusted for maternal age at enrollment (years), maternal education level ( 0 to 4 years, 5 to 7 years, 8 to 11 years, and $\geq 12$ years), marital status (married or not), maternal occupation (employed or not), household wealth index (quintiles), total energy intake (kcal/d), intervention assignment (multiple micronutrient supplementation or control), and first-trimester BMI category (underweight, normal-weight, or overweight/obese). Missing data on maternal occupation and total energy intake were accounted for by using the missing indicator method

c Computed by assigning the median concentration of each quartile to participants in the corresponding quartile as a continuous variable

3) the inverse association of CHI3L1 with inadequate GWG was stronger among underweight women than normal-weight women.

\section{Discussion}

In this observational analysis using data from a randomized controlled trial in Dar es Salaam, Tanzania, we report that higher mid-pregnancy plasma concentrations of leptin and CHI3L1 are associated with greater GWG percent adequacy in a dose-response pattern. We also find that higher mid-pregnancy plasma leptin concentrations are associated with a lower risk of inadequate GWG, while higher mid-pregnancy leptin and CHI3L1 concentrations are associated with a higher risk of excessive GWG. The associations of leptin and CHI3L1 with inadequate GWG are stronger during the second trimester than during the third trimester.
Inflammatory and angiogenic processes are involved in various aspects of maternal metabolism that may play a role in the accumulation of gestational weight. The IOM identifies the evaluation of whether maternal biomarkers can enhance clinical prediction and guide interventions for women with GWG outside the recommended ranges as an important research area [1]. However, the associations of inflammatory, angiogenic, and metabolic pathways with GWG are yet to be fully elucidated, especially in sub-Saharan Africa, which has a high burden of inadequate GWG and an emerging burden of excessive GWG $[16,17]$. This study fills this gap by examining the associations between the mid-pregnancy plasma concentrations of a wide array of potential biomarkers and GWG.

Leptin is an adipocytokine synthesized by adipose tissues and the placenta during pregnancy [42]. In the first trimester, the placenta produces large amounts of leptin 
Table 4 Mid-pregnancy plasma concentrations of leptin and chitinase-3-like protein-1 and inadequate and excessive gestational weight gain in a cohort of pregnant women in Dar es Salaam, Tanzania, 2001-2004 a,b

\begin{tabular}{|c|c|c|}
\hline & $\begin{array}{l}\text { Inadequate GWG } \\
(n=533)\end{array}$ & $\begin{array}{l}\text { Excessive GWG } \\
(n=170)\end{array}$ \\
\hline & RR $(95 \% \mathrm{CI})$ & RR $(95 \% \mathrm{Cl})$ \\
\hline \multicolumn{3}{|l|}{ Leptin } \\
\hline Quartile 1 & 1.00 (Reference) & 1.00 (Reference) \\
\hline Quartile 2 & $0.98(0.85,1.11)$ & $1.24(0.77,2.00)$ \\
\hline Quartile 3 & $0.90(0.78,1.04)$ & $1.27(0.82,1.97)$ \\
\hline Quartile 4 & $0.77(0.65,0.91)$ & $1.57(1.03,2.39)$ \\
\hline P-trend ${ }^{c}$ & 0.001 & 0.03 \\
\hline \multicolumn{3}{|l|}{ CHI3L1 } \\
\hline Quartile 1 & 1.00 (Reference) & 1.00 (Reference) \\
\hline Quartile 2 & $1.06(0.91,1.23)$ & $0.85(0.57,1.28)$ \\
\hline Quartile 3 & $0.95(0.81,1.12)$ & $1.05(0.73,1.51)$ \\
\hline Quartile 4 & $1.00(0.85,1.17)$ & $1.35(0.99,1.84)$ \\
\hline$P$-trend ${ }^{c}$ & 0.7 & 0.007 \\
\hline
\end{tabular}

${ }^{a}$ Estimates were obtained from log-binomial models. Modified Poisson models with robust variance estimation were used to handle model convergence issues whenever necessary. Inadequate and excessive gestational weight gain was defined as $<90 \%$ and $>125 \%$ percent adequacy, respectively, based on the Institute of Medicine guidelines. The reference outcome for inadequate gestational weight gain included adequate and excessive gestational weight gain; the reference outcome for excessive gestational weight gain included adequate and inadequate gestational weight gain. CHI3L1 chitinase-3-like protein-1; $C l$ confidence interval; GWG gestational weight gain; $R R$ risk ratio

${ }^{b}$ All models were adjusted for maternal age at enrollment (years), maternal education level ( 0 to 4 years, 5 to 7 years, 8 to 11 years, and $\geq 12$ years), marital status (married or not), maternal occupation (employed or not), household wealth index (quintiles), total energy intake ( $\mathrm{kcal} / \mathrm{d})$, intervention assignment (multiple micronutrient supplementation or control), and first-trimester BMI category (underweight, normal-weight, or overweight/obese). Missing data on maternal occupation and total energy intake were accounted for by using the missing indicator method

c Computed by assigning the median concentration of each quartile to participants in the corresponding quartile as a continuous variable

transferred primarily into the maternal circulation, resulting in a marked increase in maternal plasma leptin concentrations $[42,43]$. Maternal plasma leptin concentrations peak in the late second or early third trimester and stay elevated throughout the remainder of the pregnancy [44]. Leptin is also implicated in various aspects of maternal metabolism, including satiety and energy balance, trophoblast invasion (vital for the establishment of pregnancy), placental growth, nutrient transfer, and the regulation of neuroendocrine functions [45-47]. Further, leptin is involved in immune response and T-cell activation and has pro-inflammatory properties associated with adverse pregnancy outcomes, including preeclampsia, gestational diabetes, preterm birth, and intrauterine growth restriction [20,26, 42].

Findings from this study add to the emerging body of literature in high-income settings suggesting a positive association between mid-pregnancy leptin and GWG among women [48-50]. Notably, in a recent study among
Canadian women, higher second-trimester leptin concentrations were associated with greater late-pregnancy GWG [50]. The positive association between leptin and weight during pregnancy is in contrast to leptin's physiologic effects of satiety (inhibiting hunger) and diminishing fat storage among non-pregnant individuals [50]. Although there is an increased concentration of leptin in circulation during pregnancy, a positive energy balance is achieved through a decreased production of central leptin in adipose tissue and resistance to leptin's central satiety effect [51, 52]. This inverse association between leptin and inadequate GWG is also in line with the inverse association between leptin and small for gestational age previously reported in the same cohort [20], as inadequate GWG has been consistently associated with a higher risk of small for gestational age [1].

The specific biological pathways through which leptin affects GWG remain to be evaluated. Leptin may contribute to a greater accumulation of maternal body fat and placental weight during pregnancy $[42,53]$. Leptin may also represent a proxy measure of maternal insulin sensitivity, fat metabolism, or other mechanisms involved in weight gain, even if there are no direct mechanisms relating leptin per se to GWG $[1,48,54]$. Still, the consistent and strong association between mid-pregnancy leptin and late-pregnancy GWG suggests that leptin concentrations measured during mid-pregnancy may have predictive utility for the early identification of women at a higher risk of inadequate or excessive GWG, which should be evaluated in further prospective studies.

We conducted subgroup analyses to assess whether the associations of leptin and CHI3L1 with GWG may be modified by a few maternal characteristics. One notable finding is that the positive associations of leptin and CHI3L1 with GWG were stronger among overweight or obese women than underweight and normal-weight women. A previous study in Canada also finds that the positive association between second-trimester leptin concentrations and GWG is stronger in overweight women [50]. One potential explanation may be that the central satiety effect of leptin during pregnancy is weaker among overweight women $[43,55]$. Others have suggested the presence of a positive feed-forward loop that enhances the weight-increasing effect of leptin during pregnancy among overweight women [50]. It should be noted, however, that these secondary analyses on effect modification are exploratory, and any statistical interaction must be interpreted with caution. Future studies with a more substantial sample size within each BMI category (especially obesity which was rare in our study) should specifically evaluate this effect modification of leptin by early-pregnancy BMI.

CHI3L1, also known as YKL-40 or cartilage glycoprotein-39, is a glycoprotein involved in inflammation and 
extracellular matrix remodeling $[56,57]$. We found that higher mid-pregnancy plasma CHI3L1 concentrations were associated with greater weight gain during pregnancy, albeit to a smaller extent than leptin. Higher maternal plasma concentrations of CHI3L1 have been associated with a higher risk of spontaneous preterm birth in the same cohort of pregnant women [26]. However, the physiological implications of CHI3L1 in human pregnancy remain poorly understood. Future research is needed to better elucidate the role of CHI3L1 in pregnancy and perinatal outcomes.

This study provides evidence for the potential utility of biomarkers in the identification of pregnant women at high risks of gaining inadequate or excessive weight. Future work that seeks to provide early predictions of GWG may consider the values of biomarkers, especially leptin, as predictors. This study was conducted nearly two decades ago, and the prevalences of inadequate and excessive GWG may not accurately reflect the current burdens of suboptimal GWG. However, recent evidence shows that GWG in sub-Saharan Africa is extremely low and is not showing signs of improvements from the beginning of the new millennium [58]. The associations between biomarkers and GWG is unlikely to be heavily time-dependent, and our findings remain timely for future investigations into biomarkers of GWG.

The strengths of this study include the prospective design, the inclusion of an extensive panel of proteins, the use of GWG percent adequacy measures that are independent of gestational duration, and the focus on an understudied population in sub-Saharan Africa. We maintained an $\alpha$ level of 0.05 and did not account for the examination of numerous proteins or outcomes. This may have increased the probability of type I error for the composite null hypothesis that "none of the proteins were associated with GWG." However, the aim of the study was to discover potentially important biomarkers for further investigation, so quantitative adjustments for multiple testing (e.g., the Bonferroni correction) would have inflated the probability of type II error and masked potential associations [41].

This study has some potential limitations. First, prepregnancy weight was not available in this study, and for most (some 98\%) of the participants, we imputed first-trimester weight at 9 weeks as a proxy for prepregnancy weight. This may have introduced measurement errors in the GWG metrics. However, this methodological approach to imputing early-pregnancy weight based on weight measures collected later during pregnancy has previously been validated using pregnant women in Dar es Salaam, Tanzania, with good imputation accuracy [35], and any misclassification is likely nondifferential with respect to concentrations of the proteins. Second, gestational age in this study was calculated based on self-reported last menstrual period (as opposed to the gold standard of ultrasound, which was difficult to access at the time of the study), which may have introduced measurement error in gestational dating. Third, as an observational analysis, the potential of residual confounding could not be ruled out, even though we adjusted extensively for potential confounders. Finally, the proteins were measured only once (during mid-pregnancy). Future studies with repeatedly collected measures of proteins should evaluate the longitudinal associations between these proteins and GWG metrics over the course of pregnancy.

\section{Conclusions}

Leptin and potentially CHI3L1 may be associated with a greater weight gain during pregnancy among pregnant women in sub-Saharan Africa. Future studies should examine the physiological roles of these proteins in the accumulation of weight during pregnancy. Further work is also needed to evaluate the clinical predictive utility of mid-pregnancy maternal leptin concentrations in the early identification of women at high risks of inadequate and excessive GWG.

\section{Abbreviations \\ Ang-1: Angiopoietin-1; Ang-2: Angiopoietin-2; Angpt13: Angiopoietin-like 3; BMI: Body mass index; C5a: Complement component C5a; CHI3L1: Chitinase- 3-like protein-1; CRP: C-reactive protein; Factor D: Complement factor D; GA: Gestational age; GWG: Gestational weight gain; IL-18 BP: Interleukin-18 binding protein; IL1 $\beta$ : Interleukin-1 beta; IOM: Institute of Medicine; MCP-1/ CCL2: Monocyte chemoattractant protein-1; MIP $\beta / C C L 4$ : Macrophage inflam- matory protein-1 beta; MMS: Multiple micronutrient supplementation; MUAC: Mid-upper arm circumference; PGF: Placental growth factor; RCT: Randomized controlled trial; sEng: Soluble endoglin; sFlt-1: Soluble fms-like tyrosine kinase 1; sICAM1: Soluble intercellular adhesion molecule-1; STNFR2: Soluble tumor necrosis factor receptor 2; T1: First trimester; T2: Second trimester; T3: Third trimester; VEGF-A: Vascular endothelial growth factor.}

\section{Supplementary Information}

The online version contains supplementary material available at https://doi. org/10.1186/s12884-021-04146-0.

Additional file 1:.

Additional file 2:

Additional file 3:.

Additional file 4:

Additional file 5:

\section{Acknowledgements}

We thank the mothers and children, the field teams, including nurses, midwives, supervisors, the laboratory staff, the administrative staff, and all other members of the Harvard-Tanzania collaboration for making this study possible.

Authors' contributions

CRM, SA, WU, WCL, KCK and WWF conceived and designed the original research; CRM, SA, WU, ALC, and KTH contributed to laboratory analyses; DW, AMD, NP, EL, MW, and WWF developed the statistical analysis plan; DW and 
AMD performed statistical data analyses; DW drafted the work. All authors contributed to the interpretation of the results. All authors read, critically revised, and approved the final manuscript.

\section{Funding}

This work was supported by the Eunice Kennedy Shriver National Institute of Child Health and Human Development of the National Institutes of Health (NICHD R01 37701 to WWF), the Canadian Institutes of Health Research (CIHR FDN 148439 to KCK), the Canada Research Chairs program (to KCK), and the Bill and Melinda Gates Foundation (OPP1204850 to WWF). The funding sources had no involvement in study design; in the collection, analysis and interpretation of data; in the writing of the report; or in the decision to submit the article for publication.

\section{Availability of data and materials}

The datasets generated and/or analysed during the current study are not publicly available due to the need to protect the privacy of individuals that participated in the study, but are available from the corresponding author on reasonable request.

\section{Declarations}

\section{Ethics approval and consent to participate}

This study was approved by the institutional review boards at Muhimbili University of Health and Allied Sciences, the University Health Network (Toronto, Ontario, Canada), and Harvard T.H. Chan School of Public Health. All women provided written informed consent to participate. The study and all methods were carried out in accordance with the guidelines and regulations in the Declaration of Helsinki.

\section{Consent for publication}

Not applicable.

\section{Competing interests}

The authors declare that they have no competing interests.

\section{Author details}

${ }^{1}$ Department of Global Health and Population, Harvard T.H. Chan School of Public Health, Harvard University, Boston, MA 02120, USA. ${ }^{2}$ Sandra Rotman Laboratories, University Health Network, Toronto, Ontario, Canada. ${ }^{3}$ Institutional Centers for Clinical and Translational Research, Boston Children's Hospital, Boston, MA, USA. ${ }^{4}$ Department of Biostatistics, Harvard T.H. Chan School of Public Health, Harvard University, Boston, MA, USA. ${ }^{5}$ Department of Epidemiology, Harvard T.H. Chan School of Public Health, Harvard University, Boston, MA, USA. ${ }^{6}$ Department of Microbiology and Immunology, Muhimbili University of Health and Allied Sciences, Dar es Salaam, Tanzania. ${ }^{7}$ Department of Pediatrics, Ryan White Center for Pediatric Infectious Disease and Global Health, Indiana University School of Medicine, Indianapolis, Indiana, USA. ${ }^{8}$ Department of International Health, Johns Hopkins Bloomberg School of Public Health, Baltimore, MD, USA. ${ }^{9}$ Department of Medicine, University of Washington, Seattle, Washington, USA. ${ }^{10}$ Department of Medicine, University of Toronto and University Health Network, Toronto, Ontario, Canada. ${ }^{11}$ Department of Nutrition, Harvard T.H. Chan School of Public Health, Harvard University, Boston, MA, USA.

Received: 2 May 2021 Accepted: 22 September 2021

Published online: 06 October 2021

\section{References}

1. Rasmussen KM, Yaktine AL. Committee to reexamine IOM pregnancy weight guidelines. Food and nutrition board, board on children, youth and families, Institute of Medicine, National Research Council weight gain during pregnancy: reexamining the guidelines Washington, DC: National Academies Press; 2009.

2. Siega-Riz AM, Viswanathan M, Moos M-K, Deierlein A, Mumford S, Knaack J, et al. A systematic review of outcomes of maternal weight gain according to the Institute of Medicine recommendations: birthweight, fetal growth, and postpartum weight retention. Am J Obstetr Gynecol. 2009;201(4):339. e331-14.

3. Han Z, Lutsiv O, Mulla S, Rosen A, Beyene J, McDonald SD, et al. Low gestational weight gain and the risk of preterm birth and low birthweight: a systematic review and meta-analyses. Acta Obstet Gynecol Scand. 2011;90(9):935-54.

4. Goldstein RF, Abell SK, Ranasinha S, Misso M, Boyle JA, Black MH, et al. Association of gestational weight gain with maternal and infant outcomes: a systematic review and meta-analysis. Jama. 2017;317(21):2207-25.

5. Chen A, Feresu SA, Fernandez C, Rogan WJ. Maternal obesity and the risk of infant death in the United States. Epidemiology (Cambridge, Mass). 2009:20(1):74.

6. Davis RR, Hofferth SL. The association between inadequate gestational weight gain and infant mortality among US infants born in 2002. Matern Child Health J. 2012;16(1):119-24.

7. Freese KE, Himes KP, Hutcheon JA, Parisi SM, Brooks MM, McTigue K, et al. Excessive gestational weight gain is associated with severe maternal morbidity. Annals of Epidemiology. 2020;50:52-6 e51.

8. Zilko CEM, Rehkopf D, Abrams B: Association of maternal gestational weight gain with short-and long-term maternal and child health outcomes. American J Obstetr Gynecol 2010, 202(6):574. e571-574. e578.

9. Wrotniak BH, Shults J, Butts S, Stettler N. Gestational weight gain and risk of overweight in the offspring at age $7 \mathrm{y}$ in a multicenter, multiethnic cohort study. Am J Clin Nutr. 2008;87(6):1818-24.

10. Oken E, Kleinman KP, Belfort MB, Hammitt JK, Gillman MW. Associations of gestational weight gain with short-and longer-term maternal and child health outcomes. Am J Epidemiol. 2009;170(2):173-80.

11. Mamun A, Mannan M, Doi S. Gestational weight gain in relation to offspring obesity over the life course: a systematic review and bias-adjusted meta-analysis. Obes Rev. 2014;15(4):338-47.

12. Hawley NL, Johnson W, Hart CN, Triche EW, Ching JA, Muasau-Howard B, et al. Gestational weight gain among American Samoan women and its impact on delivery and infant outcomes. BMC pregnancy and childbirth. 2015;15(1):10.

13. World Health Organization: WHO recommendations on antenatal care for a positive pregnancy experience: World Health Organization; 2016.

14. Stephenson J, Heslehurst N, Hall J, Schoenaker DA, Hutchinson J, Cade JE, et al. Before the beginning: nutrition and lifestyle in the preconception period and its importance for future health. Lancet. 2018;391(10132):1830-41.

15. Coffey D. Prepregnancy body mass and weight gain during pregnancy in India and sub-Saharan Africa. Proc Natl Acad Sci. 2015;112(11):3302-7.

16. Asefa F, Cummins A, Dessie Y, Hayen A, Foureur M. Gestational weight gain and its effect on birth outcomes in sub-Saharan Africa: systematic review and meta-analysis. PLoS One. 2020;15(4):e0231889.

17. Wang D, Wang M, Darling AM, Perumal N, Liu E, Danaei G, et al. Gestational weight gain in low-income and middle-income countries: a modelling analysis using nationally representative data. BMJ Glob Health. 2020:5(11).

18. Jaacks LM, Kavle J, Perry A, Nyaku A. Programming maternal and child overweight and obesity in the context of undernutrition: current evidence and key considerations for low-and middle-income countries. Public Health Nutr. 2017;20(7):1286-96.

19. Martínez-Hortelano JA, Cavero-Redondo I, Álvarez-Bueno C, GarridoMiguel M, Soriano-Cano A, Martínez-Vizcaíno V. Monitoring gestational weight gain and prepregnancy BMI using the 2009 IOM guidelines in the global population: a systematic review and meta-analysis. BMC Pregnancy and Childbirth. 2020;20(1):1-12.

20. Darling AM, McDonald CR, Conroy AL, Hayford KT, Liles WC, Wang M, et al. Angiogenic and inflammatory biomarkers in midpregnancy and smallfor-gestational-age outcomes in Tanzania. American J Obstetr Gynecol. 2014;211(5):509 e501-509. e508.

21. Fawzi WW, Msamanga GI, Urassa W, Hertzmark E, Petraro P, Willett WC, et al. Vitamins and perinatal outcomes among HIV-negative women in Tanzania. N Engl J Med. 2007;356(14):1423-31.

22. Urassa W, Godoy K, Killewo J, Kwesigabo G, Mbakileki A, Mhalu F, et al. The accuracy of an alternative confirmatory strategy for detection of antibodies to HIV-1: experience from a regional laboratory in Kagera, Tanzania. J Clin Virol. 1999;14(1):25-9. 
23. Filmer D, Pritchett LH. Estimating wealth effects without expenditure data-or tears: an application to educational enrollments in states of India. Demography. 2001;38(1):115-32.

24. Njelekela MA, Mpembeni R, Muhihi A, Mligiliche NL, Spiegelman D, Hertzmark E, et al. Gender-related differences in the prevalence of cardiovascular disease risk factors and their correlates in urban Tanzania. BMC Cardiovasc Disord. 2009:9(1):30.

25. Changamire FT, Mwiru RS, Msamanga GI, Spiegelman D, Urassa W, Hertzmark E, et al. Macronutrient and sociodemographic determinants of gestational weight gain among HIV-negative women in Tanzania. Food Nutr Bull. 2014;35(1):43-50.

26. McDonald CR, Darling AM, Conroy AL, Tran V, Cabrera A, Liles WC, et al. Inflammatory and angiogenic factors at mid-pregnancy are associated with spontaneous preterm birth in a cohort of Tanzanian women. PLoS One. 2015;10(8):e0134619.

27. Changamire FT, Mwiru RS, Peterson KE, Msamanga Gl, Spiegelman D, Petraro $P$, et al. Effect of multivitamin supplements on weight gain during pregnancy among HIV-negative women in Tanzania. Maternal \& Child Nutrition. 2015;11(3):297-304.

28. Gibbs R, Romero R, Hillier S, Eschenbach D, Sweet RL. A review of premature birth and subclinical infection. Am J Obstet Gynecol. 1992;166(5):1515-28.

29. Steinborn A, Niederhut A, Solbach C, Hildenbrand R, Sohn C, Kaufmann M. Cytokine release from placental endothelial cells, a process associated with preterm labour in the absence of intrauterine infection. Cytokine. 1999;11(1):66-73.

30. Keelan J, Blumenstein M, Helliwell R, Sato T, Marvin K, Mitchell M. Cytokines, prostaglandins and parturition—a review. Placenta. 2003;24:S33-46.

31. Levine RJ, Maynard SE, Qian C, Lim K-H, England LJ, Yu KF, et al. Circulating angiogenic factors and the risk of preeclampsia. N Engl J Med. 2004;350(7):672-83.

32. Romero R, Gotsch F, Pineles B, Kusanovic JP. Inflammation in pregnancy: its roles in reproductive physiology, obstetrical complications, and fetal injury. Nutrition reviews. 2007;65(suppl_3):S194-202.

33. Romero R, Chaiworapongsa T, Erez O, Tarca AL, Gervasi MT, Kusanovic JP, et al. An imbalance between angiogenic and anti-angiogenic factors precedes fetal death in a subset of patients: results of a longitudinal study. J Matern Fetal Neonatal Med. 2010;23(12):1384-99.

34. Conroy AL, Silver KL, Zhong K, Rennie M, Ward P, Sarma JV, et al. Complement activation and the resulting placental vascular insufficiency drives fetal growth restriction associated with placental malaria. Cell Host Microbe. 2013;13(2):215-26

35. Yang J, Wang D, Darling AM, Liu E, Perumal N, Fawzi WW, et al. Methodological approaches to imputing early-pregnancy weight based on weight measures collected during pregnancy. BMC Med Res Methodol. 2021;21(1):1-10.

36. Md O, Onyango AW. Borghi E, Siyam a, Nishida C, Siekmann J: development of a WHO growth reference for school-aged children and adolescents. Bull World Health Organ. 2007;85:660-7.

37. Adu-Afarwuah S, Lartey A, Okronipa H, Ashorn P, Ashorn U, Zeilani M, et al. Maternal supplementation with small-quantity lipid-based nutrient supplements compared with multiple micronutrients, but not with Iron and folic acid, reduces the prevalence of low gestational weight gain in semiurban Ghana: a randomized controlled trial. J Nutr. 2017;147(4):697-705

38. Zou G. A modified poisson regression approach to prospective studies with binary data. Am J Epidemiol. 2004;159(7):702-6.

39. Spiegelman D, Hertzmark E. Easy SAS calculations for risk or prevalence ratios and differences. Am J Epidemiol. 2005;162(3):199-200.

40. World Health Organization: Nutritional anaemias: tools for effective prevention and control. 2017.

41. Rothman KJ. No adjustments are needed for multiple comparisons. Epidemiology. 1990:43-6.

42. Henson MC, Castracane VD. Leptin in pregnancy: an update. Biol Reprod. 2006;74(2):218-29.
43. Hauguel-de Mouzon S, Lepercq J, Catalano P. The known and unknown of leptin in pregnancy. Am J Obstet Gynecol. 2006;194(6):1537-45.

44. Tessier D, Ferraro Z, Gruslin A. Role of leptin in pregnancy: consequences of maternal obesity. Placenta. 2013;34(3):205-11.

45. Mantzoros CS. Role of leptin in reproduction. Ann N Y Acad Sci. 2000;900(1):174-83

46. Briana DD, Malamitsi-Puchner A. Adipocytokines in normal and complicated pregnancies. Reprod Sci. 2009;16(10):921-37.

47. Molvarec A, Szarka A, Walentin S, Bekő G, Karádi I, Prohászka Z, et al. Serum leptin levels in relation to circulating cytokines, chemokines, adhesion molecules and angiogenic factors in normal pregnancy and preeclampsia. Reprod Biol Endocrinol. 2011;9(1):1-9.

48. Stein TP, Scholl TO, Schluter MD, Schroeder CM. Plasma leptin influences gestational weight gain and postpartum weight retention. Am J Clin Nutr. 1998;68(6):1236-40.

49. Walsh JM, McGowan CA, Mahony RM, Foley ME, McAuliffe FM. Obstetric and metabolic implications of excessive gestational weight gain in pregnancy. Obesity. 2014;22(7):1594-600.

50. Lacroix M, Battista M-C, Doyon M, Moreau J, Patenaude J, Guillemette $L$, et al. Higher maternal leptin levels at second trimester are associated with subsequent greater gestational weight gain in late pregnancy. BMC Pregnancy Childbirth. 2016;16(1):1-9.

51. Page-Wilson G, Reitman-Ivashkov E, Meece K, White A, Rosenbaum M, Smiley RM, et al. Cerebrospinal fluid levels of leptin, proopiomelanocortin, and agouti-related protein in human pregnancy: evidence for leptin resistance. J Clin Endocrinology Metab. 2013;98(1):264-71.

52. Sámano R, Martínez-Rojano H, Chico-Barba G, Godínez-Martínez E, Sánchez-Jiménez B, Montiel-Ojeda D, et al. Serum concentration of leptin in pregnant adolescents correlated with gestational weight gain, postpartum weight retention and newborn weight/length. Nutrients. 2017:9(10):1067.

53. Highman TJ, Friedman JE, Huston LP, Wong WW, Catalano PM. Longitudinal changes in maternal serum leptin concentrations, body composition, and resting metabolic rate in pregnancy. Am J Obstet Gynecol. 1998;178(5):1010-5.

54. Okereke NC, Huston-Presley L, Amini SB, Kalhan S, Catalano PM. Longitudinal changes in energy expenditure and body composition in obese women with normal and impaired glucose tolerance. American J Physiology-Endocrinology and Metabolism. 2004;287(3):E472-9.

55. Grattan DR, Ladyman SR, Augustine RA. Hormonal induction of leptin resistance during pregnancy. Physiol Behav. 2007;91(4):366-74.

56. Ober C, Chupp GL. The chitinase and chitinase-like proteins: a review of genetic and functional studies in asthma and immune-mediated diseases. Curr Opin Allergy Clin Immunol. 2009;9(5):401.

57. Lee CG, Da Silva CA, Dela Cruz CS, Ahangari F, Ma B, Kang M-J, et al. Role of chitin and chitinase/chitinase-like proteins in inflammation, tissue remodeling, and injury. Annu Rev Physiol. 2011;73:479-501.

58. Gebremedhin S, Bekele T. Gestational weight gain in sub-Saharan Africa: estimation based on pseudo-cohort design. PLoS One 2021;16(5):e0252247.

\section{Publisher's Note}

Springer Nature remains neutral with regard to jurisdictional claims in published maps and institutional affiliations.

Ready to submit your research? Choose BMC and benefit from:

- fast, convenient online submission

- thorough peer review by experienced researchers in your field

- rapid publication on acceptance

- support for research data, including large and complex data types

- gold Open Access which fosters wider collaboration and increased citations

- maximum visibility for your research: over 100M website views per year

At $\mathrm{BMC}$, research is always in progress.

Learn more biomedcentral.com/submissions 